\title{
DE LA ILUSIÓN A LA FRUSTRACIÓN: LAS CANDIDATURAS INDEPENDIENTES EN EL MUNICIPIO DE JUÁREZ
}

\author{
NOLBERTO ACOSTA \\ El partido es la locura de muchos para la ganancia de pocos \\ (Jonathan Swift)
}

\section{RESUMEN}

$\amalg$ 1 fenómeno de las candidaturas independientes tiene que ver con el agotamiento de los partidos políticos, con la necesidad de oxigenar el sistema de partidos, con la flexibilización de las leyes electorales que permitan esta modalidad de participación y con el manejo hegemónico del sistema político mexicano. Es una práctica que inició a finales del siglo xx en diferentes partes del mundo y que se incluyó en ámbito políticoelectoral mexicano en 2015. Conforme se vienen dando procesos electorales en las diferentes regiones del país, se incorpora a las legislaciones locales, para el caso de Chihuahua, se hacen las modificaciones a contracorriente casi al iniciar el proceso electoral vigente para la renovación de los dos poderes legislativo y ejecutivo estatal, así como de ayuntamiento. De siete planillas que fueron autorizadas en las primarias, solo una pudo acceder a la categoría de candidatura, el resto se quedó en la precandidatura, 
debido al diseño legal que hicieran los partidos políticos para obstruir su participación.

Palabras clave: Candidaturas, independientes, ayuntamiento, partidos políticos.

\section{ANTECEDENTES}

En 2006, Crespo ${ }^{1}$ decía que varios países democráticos en el mundo contemplaban las candidaturas independientes en sus sistemas electorales, entre estos países estaban Estados Unidos, Inglaterra, Japón, Alemania, Italia, Portugal, España, Suiza, Bélgica, Gran Bretaña, Irlanda, India, Holanda, Dinamarca, Canadá y Finlandia. Asimismo, menciona que también democracias incipientes como Albania, Armenia, Bolivia, Chile Rumania, Rusia y Venezuela, entre otras, desarrollan procesos electorales bajo esta modalidad. En un estudio sobre el tema, Chanona² dice que en el mundo existe $36 \%$ de países, equivalente a 81 naciones que están de acuerdo y permiten las candidaturas independientes, para el registro de las candidaturas, se establecen varias modalidades: a) claramente especificado el número de firmas que requieren, b) especifica con claridad un depósito económico que garantice la viabilidad, c) no está claro el requisito de registro, d) otras modalidades y e) no aplica.

Las excepciones en América Latina se dan en Brasil, Costa Rica y el Salvador (Córdova; 2012), ${ }^{3}$ este mismo autor plantea, la posibilidad que existe en países como Colombia, Honduras, Perú, República Dominicana y Venezuela, pero con la modalidad de que dichas candidaturas independientes no sean exclusivamente dirigidas a ciudadanos, sino, también a agrupaciones y movimientos independientes a los partidos políticos. La presencia de las candidaturas independientes en el mundo es aún muy marginal y, se ha focalizado en cargos más relacionados con la exaltación

1 http://democraciamexico.blogspot.mx/2006/candidaturas-independientes-enel.html (Consultado el marzo 5 de 2016).

2 http://www.fusda.org/Revista 13-2AlejandroChanona.pdf (Consultado el marzo 5 de 2016).

3 http://www.adnpolitico.com/opinion/2012/analisis-una-mirada-a-las-candidaturas-independientes (Consultada el 6 de marzo de 2016). 
de la figura pública. Los espacios destinados a la representación legislativa siguen siendo ocupados por los grandes partidos políticos que definen el rumbo del país o de la entidad determinada.

El caso más emblemático por su influencia y cercanía con México, se dio en Estados Unidos, con la candidatura de Henry Ross Perot en 1992. Este millonario texano logró darle un susto al tradicional bipartidismo -Partido Republicano y Partido Demócrata- que intermitentemente ocupan la inmensa mayoría en el Congreso y siempre, en la presidencia de la nación. Perot, logró la nada despreciable cantidad de 19743821 votos, equivalente a un $18.9 \%$ del voto popular, aún, cuando no obtuvo ningún voto electoral -condición indispensable para poder gobernar-, ${ }^{4}$ logró darle un estrujón al sistema bipartidista.

En el mismo plano internacional, está el movimiento político español Podemos, ${ }^{5}$ que aunque en corto plazo se convirtió en partido político por la forma en que surgió y se consolidó, alentó las candidaturas independientes en México. ${ }^{6}$ Podemos inició como un movimiento integrado por un grupo de intelectuales de diferentes áreas, pero comprometidos con impulsar políticas públicas orientadas a la izquierda, su planteamiento "mover ficha", un documento-manifiesto que planteó convertir la indignación social en un cambio político, le valió que en menos de 24 horas lograría los 50 mil "me gusta" que se habían propuesto para participar en el proceso electoral que se avecinaba.

\footnotetext{
4 Las elecciones en Estados Unidos para elegir presidente de la nación se ganan con un total de 270 votos electorales, de un total de 538 que dan la suma de las 50 entidades. En este país, cada estado tiene un número de votos electorales proporcionales a la población, por ejemplo, California cuenta con 55, es el que mayor cantidad de votos electorales tiene; en tanto, hay varias entidades federativas que cuentan con apenas 3 votos electorales. Un voto electoral, es el equivalente a un distrito, y el que gane más distritos en el estado, gana todo el estado (http://www. archives.gov/federal-register/electoral-college/faq.html, consultada el 4 de marzo de 2016).

5 http://podemos.info/Podemos.info (Consultada el 7 de marzo de 2016).

6 Pedro Kumamoto en su visita a Ciudad Juárez en agosto de 2015 comentó la influencia de este recién constituido partido político español.
} 


\section{MÉXICO Y SU PROCESO HISTÓRICO-POLÍTICO}

El desarrollo de la vida política en México ha estado marcada una alta dosis de autoritarismo, con el fin del porfiriato terminó con una época dominada por un gobierno altamente centralizado, muy parecido a las viejas monarquías de origen colonial. En tanto que la Revolución mexicana, trajo por su parte una alta inestabilidad gubernamental, con semejanzas en este aspecto al que se dio entre la independencia y la reforma. Fue hasta el Maximato, con la instauración del Partido Nacional Revolucionario (PNR) que el país se "asilenció", aún, con lo que significó la guerra cristera.

El régimen presidencialista sirvió para acabar con las conspiraciones del legislativo, del ejército y del clero [...], el partido predominante sirvió para acabar con los caudillos y sus partidos de membrete [...] el régimen centralista sirvió para acabar con los feudos regionales (González Casanova, 1975: p. 86).

Ya con el gobierno de Lázaro Cárdenas, se radicaliza el ideal surgida de la Revolución y se incorporan algunas reformas sociales al proyecto de gobierno. Pero, también se corporativiza la organización gremial y se transforma el PNR en Partido de la Revolución Mexicana (PRM) órgano político encargado de llevar la ideología y la acción de gobierno.

Hasta 1946, la Ley Electoral no había sufrido grandes trasformaciones en cuanto al manejo del ideario político para la organización y funcionamiento de la conformación de gobierno. Con la transformación del PRM en Partido Revolucionario Institucional (PRI), se promulga la Ley Electoral,? misma que dará personalidad jurídica a los partidos políticos, asimismo, les otorgará el monopolio para participar electoralmente en la conformación de los gobiernos -poder ejecutivo y poder legislativo- federal y locales. También, es pertinente apuntar que la organización y vigilancia de los procesos electorales quedaron en manos de comisiones creadas desde el gobierno (Rodríguez, 1989).

7 http://www.diputados.gob.mx/museo/s_nues8.htm (Consultada el 12 de marzo de 2016). 
Las candidaturas independientes, ciudadanas o al margen de los partidos políticos tenían cabida en la legislación electoral; la Constitución Política Mexicana no hacía alusión a los partidos políticos, la referencia para participar electoralmente refería a la libertad que tenían los ciudadanos de asociarse para competir por los puestos de elección popular. En este sentido, se podría decir que las candidaturas ciudadanas eran permitidas. Por otra parte, está documentada una gran cantidad de partidos políticos regionales a lo largo y ancho del territorio, muchos de ellos ligados a jefes revolucionarios regionales (Fuentes, 1969).

La presencia de los partidos políticos era sumamente precaria; por una parte, el Partido Acción Nacional (PAN) surgido en 1939 había obtenido algunos triunfos regionales, principalmente en Michoacán. Según Valdez (1999: 104) la reforma a la legislación electoral en 1963 al crearse los diputados de partido hicieron más visible al PAN y pasa de ser una "oposición leal a una oposición real". Valdez, dice que el tema que agarró el pan, fue el de la democracia, con ello, se entiende la lucha por la democratización y la lucha contra el monopolio del partido hegemónico.

En el terreno político-electoral se constituye un escenario que gira en torno al monopolio de los partidos políticos para la conformación de los poderes, que encaja en la tipología de Sartori (2002) y es considerado como un sistema de partido hegemónico. Bajo esta condición, el gobierno, se convirtió en el promotor de la ideología de la Revolución mexicana y llevaron a cuestas un sistema político antidemocrático que se evidenció en 1976 con la elección de José López Portillo como presidente de México, sin que hubiera competencia, es decir, fue candidato único (Campos, 2014). El PRI-gobierno, se encargaba de mantener el dominio político-electoral en todos los procesos encaminados a la renovación de poderes públicos regionales o nacionales.

El sistema de partido hegemónico, concluye a la mitad del gobierno de Ernesto Zedillo (1997), precisamente cuando el PRI pierde la mayoría en el Congreso. Y, tres años después, en el proceso electoral del 2000 triunfa el candidato del PAN, inaugurando con ello una nueva etapa de alternancia política-electoral. El gobierno panista se agotó rápidamente, el siguiente proceso electoral 
de 2006 fue el más cerrado en la historia electoral del país, la izquierda se convirtió en la opción electoral, sin embargo, con la intervención del poder presidencial y algunas artimañas le cerraron el paso. En 2012, el PAN pierde la presidencia ante el PRI, y, se dan muestras de agotamiento del sistema de partidos.

A partir del proceso electoral del 2012, se aceleran las consultas para realizar una reforma electoral que incluya las candidaturas independientes, mismas que quedaron integradas en la Ley General de Instituciones y Procedimientos Electorales aprobada en 2014. También, se cambió el nombre del Instituto Federal Electoral (IFE), por el de Instituto Nacional Electoral (INE). El marco jurídico de la Ley electoral federal, no necesariamente se ha bajado en condiciones similares en las leyes locales. En el caso de las candidaturas independientes se dan marcadas diferencias entre lo local y lo nacional, por lo menos en Chihuahua, así se ha presentado.

\section{CANDIDATURAS INDEPENDIENTES CHIHUAHUA}

La convocatoria estatal de Chihuahua para las candidaturas independientes fue emitida con toda la intención de obstaculizar la compartición del poder con representantes populares surgidos por esta vía: a) en primera instancia, se emitió con fechas muy forzadas, lo que impediría a varios candidatos poder inscribirse; b) el porcentaje de firmas requeridas para obtener el registro fue superior al estipulado en la Ley Electoral Federal; c) el porcentaje de votos por sección electoral, así como de secciones cubiertas también estaba en la lógica que obstaculizará la participación; siendo $2 \%$ y más de $50 \%$ respectivamente, d) se impedía que candidatos con menos de tres años de militancia en un partido político pudieran participar como independientes. Grosso modo, esos eran algunos de puntos surgidos del Congreso y con toda la intención de inhibir lo recientemente ocurrido en Nuevo León, se le denominó, "Ley anti-Bronco", por considerarla como un experimento que lograra controlar ciertas variables que impidieran al PRI perder electoralmente la entidad.

La Reforma Electoral realizada por los partidos políticos, en torno a las candidaturas independientes, permite que estos con- 
tinúen disfrutando del monopolio del poder y del disfrute del mismo, pues, en la entidad se fortalece la partidocracia. Con esta reforma, lejos de acotar la fuerza de los partidos políticos en los órganos de poder institucional, refuerza su presencia y de paso, beneficia a los "pequeños" partidos para que logren obtener el porcentaje requerido. En el caso concreto de Chihuahua, a diferencia de lo que establece la legislación electoral federal, aquí, no se requiere un $3 \%$ de los sufragios válidos para tener derecho a la conservación del registro y por ende, al reparto de cargos de representación proporcional. En la Ley Electoral de Chihuahua, basta con que los partidos políticos logren 2 \% de la votación válida emitida, para que consigan la conservación del registro local.

En el artículo 191 inciso b se establece que únicamente los partidos políticos y coaliciones tendrán derecho a la asignación de regidores por el principio de representación proporcional, siempre y cuando hayan obtenido por lo menos el $2 \%$ de la votación municipal válida emitida: "La votación municipal válida emitida resultará de deducir de la votación municipal total emitida, los votos nulos y los votos a favor de candidatos independientes y candidatos no registrados. Para lo anterior, se entiende por votación municipal total emitida el total de votos depositados en las urnas de la elección municipal de ayuntamiento que corresponda".

Una interpretación ad hoc, que favorece el porcentaje de los partidos políticos y por ende, la cantidad de "representantes" populares por esta vía. Es una manipulación irreal, con ello se desconoce y se anula el voto ciudadano. En el supuesto caso de una elección competida entre un candidato independiente y un candidato de un partido político, al final de la jornada, el candidato del partido político obtiene el triunfo por un voto. Para efectos de la distribución de las posiciones de representación proporcional, el candidato o la planilla independiente no solo no obtiene nada, sino que ayuda a que los pírricos resultados del resto de los partidos políticos se incremente, por la anulación de los votos obtenidos por los candidatos independientes, se toman en cuenta solamente los votos obtenidos entre los partidos políticos.

Lo anterior, es un acto deliberado, que lleva toda la intención, no de castigar y hacer más responsables a los partidos políticos, 
sino, que por el contrario, es premio, un bono extra que les permite tener mayor presencia en la entidad. En el artículo 254, se instituye que para efectos de determinar la votación emitida, no serán contabilizados los votos recibidos a favor de candidatos independientes.

El proceso por el que deberán transitar las candidaturas independientes en la entidad queda bajo el siguiente orden, según el artículo 199. El proceso de selección de candidatos independientes comprende las etapas siguientes:
a) De la convocatoria
b) De los actos previos al registro de candidatos independientes
c) De la obtención del apoyo ciudadano y
d) Del registro de candidatos independientes.

Sobre el primer apartado, referente a la convocatoria, se establecen una serie de pasos que deben cumplirse escrupulosamente, entre ellos, entregar un oficio al órgano electoral respectivo, donde queda de manifiesto la intención de participar por el cargo respectivo. Lo más difícil de conseguir para este primer momento de "aspirante" y poder lograr ese estatus, requiere de la formación de una asociación civil, constituida legalmente ante notario público y cuyo costo oscila entre los 12 y 15 mil pesos moneda nacional.

\section{LOS PRECANDIDATOS/AS INDEPENDIENTES PARA EL AYUNTAMIENTO DE CIUDAD JUÁREZ}

Para el Ayuntamiento de Ciudad Juárez se inscribieron siete planillas, dos más para participar por distritos electorales locales con cabecera en el municipio.

- Javier Meléndez Cardona

- Arturo José Valenzuela Zorrilla

- Héctor Armando Cabada Alvídrez

- Edna Lorena Fuerte

- Antonia Hinojos Hernández

- Andrés Carbajal Casas 
- Alejandro Ramírez Guerrero

En los medios de comunicación se mencionaba que tal vez serían 12 las planillas registradas para contender por el Ayuntamiento de Juárez, finalmente solicitaron siete planillas, todas ellas cumpliendo con los requisitos, entre ellos la constitución de una asociación civil. Cabe hacer mención, que previo a que saliera la convocatoria, se estuvieron realizando encuentros por personas de la sociedad civil que tenían la intención de constituir una planilla única que enfrentara al histórico bipartidismo que se da en la ciudad. También, se dieron encuentros entre algunos de los líderes "activos" que suelen ser protagonistas en la vida pública de la ciudad. Sin embargo, ninguna de estas reuniones logró consolidar una propuesta unificada, algunos de ellos, se inscribieron de manera independiente, cada quien por su cuenta y con su planilla.

El día del registro de los suspirantes, se dieron algunas sorpresas. Por una parte, nadie esperaba que alguien pretendiera disputar el voto duro del PRI y en ese sentido, la precandidatura al ayuntamiento, encabezada por Armando Cabada, sorprendió a propios y extraños. Tampoco se esperaba una precandidatura como la encabezada por Alejandro Ramírez Guerrero, un expanista, exparticipante recientemente en la conformación del frustrado Partido Humanista. Asimismo sorprendió la candidatura de "Toñita", saltar de la huelga obrera en que estaba participando, a inscribirse en una planilla para Ayuntamiento, ¡vaya que fue de llamar la atención!

De las planillas para Ayuntamiento que entraron en esta primera etapa, la integrada por Antonia Hinojos "Toñita” y un grupo de operadoras/es de producción que habían encabezado un movimiento huelguístico en varias maquiladoras de la frontera y que pretendía llevar su movimiento de la lucha obrera a la lucha electoral, sortear el primer obstáculo no fue sencillo

Los requisitos "vaya" estaban muy duros porque es muy difícil que un obrero junte los 15 mil que piden para la inscripción, la asociación civil, todos los requisitos que pidieron eran en contra de nuestras po- 
sibilidades porque todos somos obreros despedidos, sí, entonces recibimos mucho apoyo de nuestra asesora legal licenciada Prieto, de la asamblea, de nuestro compañero Julián Contreras, otras personas que nos han apoyado económicamente, pero de fuera (Antonia Hinojos “Toñita”, entrevista).

Ningún otro/a contendiente por planillas para el Ayuntamiento manifestó haber tenido dificultad económica para cumplir con este requisito. La fórmula del cuarto distrito local, también, enfrentó esa dificultad, lograron reunir los recursos para la conformación de la asociación civil, recurriendo a las "redes de confianza" de que disponían, ya que el proyecto era parte de un grupo que trabaja en la sociedad civil organizada, cuestiones de índole cultural. En el caso del médico Valenzuela, exdirigente de la "mesa de seguridad", ${ }^{8}$ no hubo ninguna dificultad de tipo económica, además la planilla estaba conformada por ex dirigentes políticos, académicos y comerciantes, la dificultad que enfrentaba el médico, tenía relación con el origen de su nacimiento.

Yo nací en Canadá y como buen juarense, buen mexicano corporalmente, saqué un acta de aquí que decía que había nacido en Zaragoza y con eso estuve toda mi vida, pero no era un acta derecha, es la verdad, me dijeron, no digas así, di que fue un error. No cuenta, no cuenta allí, yo no era mayor de edad, pero convenimos que eso iba a reducir mis gastos universitarios en Guadalajara y así fue, esa es la verdad. Yo viví feliz aquí con un acta chueca hasta que vi la oportunidad de agradecerle a la sociedad y hacer algo bien derecho bien derecho. Pero dije, yo no puedo entrar así, porque me van a buscar hasta por debajo de la lengua [...] les dije que buscaran otro y finalmente me dijeron en la "mesa de seguridad" que nada más quedaba yo, les dije de mi problema y me dijeron que eso se arreglaba. Fue un cuete, arreglar y fue hasta el último día que podíamos entregar el papelito ante el INE, que pude cumplir (Arturo Valenzuela, encuentro con Ciudadanos Vigilantes, 25/02/2016).

8 instrumento implementado por la cúpula empresarial local para darle seguimiento a la problemática relacionada con la inseguridad de la ciudad 
El resto de los precandidatos/as no manifestó en ningún medio o entrevista haber tenido mayores problemas para cumplir con este primer apartado de requisitos. Pero si se dio una crítica generalizada a la Ley electoral estatal, hay el ánimo de participar "Este es un momento muy significativo, porque a pesar de todos los candados que la Ley Electoral de Chihuahua le pone a la candidaturas independientes, hoy se abre la posibilidad, con un trabajo constante y de mucho tiempo atrás, de poder aspirar a la candidatura de la Presidencia Municipal de nuestro querido Juaritos" (Edna Lorena Fuerte). ${ }^{9}$

El 6 de febrero de 2016 el Consejo Estatal del Instituto Estatal Electoral (IEE) de Chihuahua, publica la aceptación de las siete precandidaturas al Ayuntamiento de Ciudad Juárez y dos para los distritos 04 y 05 del mismo municipio.

\section{¿POR QUÉ PARTICIPAR POR UNA CANDIDATURA INDEPENDIENTE?}

La ausencia de políticas públicas y proyectos que impacten en el beneficio de las mayorías de la población en el conjunto del país y lógicamente en cada una de las regiones, así como las evidentes muestras de corrupción y nepotismo, han hecho evidente el agotamiento de los partidos políticos. Al modificarse la Ley Electoral que le daba el monopolio de la representación a estos institutos políticos, generalmente de carácter y presencia nacional, surgen interesados en aprovechar ese espacio que se abre para que ciudadanos sin partido político puedan contender por esos cargos de representación popular.

Cuando vimos que los partidos políticos responden a intereses meramente de ellos y a veces de grupos de poder económico, me di cuenta que la alternativa no estaba a través de los partidos, porque los gobernantes llegan prácticamente maniatados, obedecen a las directrices de los partidos políticos y gobiernan. Los presidentes municipales son encargados del despacho para los asuntos que de-

9 http://timingpolitico.com/archivos/4713. Consultado el 25 de febrero de 2016. 
terminan los intereses económicos o poder de la ciudad. Cuando fui presidente seccional de Samalayuca, me di cuenta que el poder sirve para servir a la gente, que el poder sirve para mucho si hay un gobierno sensible que vea por los intereses de la gente y no por sus partidos y por los grupos de interés que los ponen allí (Javier Meléndez, 22 de febrero de 2016).

Porque desde el año pasado que empezamos en el movimiento de la lucha con los trabajadores por mejoras salariales, por mejores condiciones en el interés de la industria maquiladora, mejor trato al obrero, y desde que empezamos el año pasado, para nada, no hemos tenido apoyo del gobierno, a nosotros no nos han difundido la problemática, nosotros no hemos tenido el apoyo de medios de comunicación independientes, no los locales, nunca nos dieron apoyo. Y a través de este movimiento nació este proyecto, verdad. Mucha gente nos ha dicho que es una ocurrencia, iqué linda ocurrencia!, porque estamos aprendiendo mucho, soy "Toñita, la obrera" que con este proyecto pretendemos llevar la problemática de la clase obrera de la industria maquiladora de Ciudad Juárez a la Presidencia municipal (Antonia Hinojos, 23 de febrero de 2016).

Yo tengo ocho años sin participar en los partidos políticos y hace más de tres años intentamos participar por el Ayuntamiento, incluso, hablamos con algunos partidos políticos, pero las cosas no se dieron porque desde el gobierno estatal no quisieron. Ahora, la Ley lo permite y es la oportunidad para participar. En este momento, soy suspirante, aspirante seré hasta que lleve todos los papeles y órgano electoral los acepte, entonces paso a la siguiente categoría de aspirante y al juntar las firmas y cumplir los requisitos, hasta entonces te vuelves candidata independiente (Edna Lorena Fuerte, Noticias Televisa, 4 de febrero de 2016).

Porque quiero una mejor ciudad donde vivir, porque quiero una mejor ciudad para todos, porque una ciudad bonita funcional y con seguridad solo se puede conseguir con funcionarios eficientes y honestos de lo que hemos escaseado mucho (Armando Cabada, 27 de enero de 2016, Canal 44).

Soy administradora pública, por una parte para mí es importante realizar acciones, pero, no es una candidatura de Edna Lorena Fuerte, es una candidatura que viene avalada por un proyecto de muchos 
años, un proyecto que significa ideas y un concepto de plan para la ciudad, diferente al que tenemos hasta ahora, enriquecido por este tiempo en el que seguramente se va modificar, se va enriquecer y se va ampliar, nosotros sí respondemos a un esquema de un nuevo trayecto para la ciudad (Edna Lorena Fuerte, Televisa, 4 de febrero de 2016).

Una administración del ayuntamiento no difiere mucho de una administración del hogar, porque una obrera puede, pudo administrar un salario de miseria para crecer, mantener a sus hijos y pagar su casa de hipoteca, si yo pude mantenerme, mi casa, mis hijos y yo con un salario de miseria, por qué no. A lo mejor creen que no lo sé, yo solo sé que no sé nada pero quiero aprender, implica mucha responsabilidad, pero estamos dispuestos a aprender. Aparte, si los que son estudiados tienen sus asesores, por qué no los puedo tener yo (Antonia Hinojos, 23 de febrero de 2016).

A excepción de "Toñita" el resto de los candidatos tenían una militancia en uno o varios partidos políticos, en el caso del médico Valenzuela, él no había estado afiliado a ningún partido político, aunque su madre hubiera sido regidora del PAN. Un caso similar es el de Armando Cabada, su familia ha ocupado cargos en las administraciones priístas y han contendido por puestos de elección popular. Edna Lorena Fuerte, Javier Meléndez, Alejandro Ramírez y Andrés Carbajal, han tenido más de una militancia. En ese sentido, muchas de las críticas de los dirigentes de los partidos políticos cuestionaban la calidad de "independientes" y los llamaban, más bien, "chapulines".

\section{LA DIFÍCIL RUTA PARA LA OBTENCIÓN DEL APOYO CIUDADANO}

Adicionalmente al cumplimiento de lo establecido en el artículo 199, deben de ser extremadamente cuidadosos de no caer en lo que se puede interpretar como un acto anticipado de campaña y con ello perder el derecho a participar, se establece una especie de "Ley Mordaza", donde no pueden comunicar a la ciudadanía por qué estarán pidiendo su firma, "la Ley Electoral de Chihuahua es muy limitante, es muy censuradora yo les voy a pedir su consideración, hay cosas que no me permiten decir, pero que las 
habré de decir, para eso me las gasto solo" (Armando Cabada, 27 de enero de 2016, Canal 44).

Las planillas y fórmulas aprobadas por el órgano electoral tienen del 7 de febrero al 7 de marzo para recabar 29 mil 322 firmas para el Ayuntamiento, estas bajo los criterios contemplados en la Ley Electoral del Estado. El conjunto de las y los precandidatos/ as necesita reunir entre todos, algo así como 205 mil 254 rúbricas de empadronados en el municipio, lo cual no es cualquier cosa, si se considera que la votación emitida en el ayuntamiento en 2013 fue de 304667 votos (IEE Chihuahua). Lo que se pide a los precandidatos independientes para entrar en la categoría de candidatos, equivale a dos terceras partes de la votación de un proceso electoral que conlleva campañas con promoción de propuestas, entrega de dadivas y todo lo que implica la elección como tal.

La verdad, es una "valentonada" entrar a un proceso al que van con la ciudadanía y sin poder decir "gran cosa" sobre su proyecto de gobierno, sin poder hacer propaganda en ningún medio de comunicación, ni siquiera pueden "ofrecer" nada, lo único de que disponen es de su palabra y que la ciudadanía en acto de buena fe les firme, prácticamente a ciegas. Tal vez por el descredito de los partidos políticos, por la corrupción imperante o por la esperanza de que se encienda una pequeña luz al final del camino, pero, la respuesta de la ciudadanía en el arranque se puede considerar como de excelente.

El domingo 7 de febrero, el centro de la ciudad lucía con gran actividad y efervescencia política. En el Kiosco de la Plaza de Armas estaba Armando Cabada con varias mesas activas y con líneas de ciudadanos/as para firmar por él, inmediatamente se percibía la presencia mediática de alguien que había estado en la pantalla de la televisora más vista de la frontera por 27 años, frente a las mesas receptoras había un espectacular móvil promoviendo su precandidatura.

A escasos cien metros de la Plaza de Armas se encontraba a la misma hora el médico Valenzuela, sonriente y jovial recababa, junto a sus compañeros de planilla, las firmas, que no eran tan abundantes como las de Armando Cabada, pero fluían intermi- 
tentemente, ellos estaban a un costado del símbolo JRZ, sobre la 16 de septiembre, así estuvieron por varias horas.

Un poco más adelante, frente a la ex Aduana, estaba Javier Meléndez amenizado por un conjunto de música norteña y con un arranque al estilo de los tradicionales procesos electorales, en su primer día, obtuvo una buena cosecha de firmantes, se podría decir, que fue lo mejor del proceso para él, quizá ello se interpretó como una señal de que las cosas iban a ser mucho más sencillas de lo que se tornaron en los próximos días.

Del resto de los aspirantes a las candidaturas independientes, Edna Lorena Fuerte declaró que "desde muy temprano de este domingo tuvimos una reunión de trabajo y nos dispusimos a trabajar directo en territorio, los cuales ya teníamos ubicados y trabajamos directamente sección por sección, tal como dice la ley que se deben recabar las firmas" (http://timingpolitico.com/ archivos/4864, consultado el 26 de febrero 2016). Mientras que Antonia Hinojos "Toñita" inicio en "las segundas" de San Francisco, para después recorrer la colonia María Martínez (http://timingpolitico.com/archivos/4864, consultado el 26 de febrero de 2016).

En términos generales, se puede decir que el inicio de la colecta de firmas fue bueno, generó bastante optimismo entre la mayoría de los participantes. Armando Cabada, declaró haber recolectado más de 8 mil firmas el primer día (Contacto 44, 8 de febrero de 2016), además dijo:

La verdad es que lo que encontramos en las calles y en las tiendas Smart, ${ }^{10}$ es una respuesta increíble por parte de la gente que nos está apoyando, nos reportan en todas las tiendas y en todos los sitios donde están nuestras mesas receptoras, que son filas de personas y que se esperan pacientemente para darnos los datos de la credencial de elector y ofrecernos la firma (http://www.oem.com.mx/elmexicano/ notas/n4074643.htm, consultado el 27 de febrero de 2016.

De acuerdo al artículo 203 que se refiere a la obtención del apoyo ciudadano, se establece en el apartado c que los aspirantes

10 Cadena de supermercados ubicados en todas las zonas de la ciudad. 
a candidatos independientes para cargos del Miembro del Ayuntamiento y sindico, contarán con treinta días hábiles, para recabar el 3 \% del padrón municipal de electores. En el caso del municipio de Ciudad Juárez que cuenta con un padrón de 977 mil 400 electores, lo que en términos más o menos relativos, tendría que conseguir aproximadamente 35 mil firmas para quedar sobrados por las que pudieran anularse.

Durante el proceso, se veían con regularidad mesas receptoras en el centro de la ciudad de las planillas "familias unidas por Juárez" encabezada por Javier Meléndez; "Toñita” de Antonia Hinojos, Médico Arturo Valenzuela de la planilla "Unidad para el desarrollo de los ciudadanos"; y Armando Cabada, con su planilla "Así las cosas, Armando”. También, habían desplegado una campaña en páginas de redes sociales y páginas personales. En el caso de "toñita" se implementó una brigada en la Universidad Autónoma de Ciudad Juárez (UACJ); Cabada a las afueras de la cadena de tiendas Smart; a Meléndez con un grupo musical en el centro y con tuits sobre la ubicación itinerante de las mesas receptoras; Valenzuela, afuera de clínicas locales. En general, estos candidatos fueron los que tuvieron una mayor actividad mediática.

Se hacían obvias las diferencias en cuanto el equipamiento y los módulos de las diferentes planillas, en el caso de Valenzuela, Meléndez y Antonia, se obtenían las copias de la credencial de elector con un celular, mientras que Cabada, contaba con dos o tres equipos de fotocopiado, un mostrador bien equipado y lucía con legajos de formatos llenos. Las mantas de apoyo, los distintivos y las ropas también marcaban diferencias, Cabada en primer orden, Valenzuela en segundo lugar, seguido de Meléndez y, finalmente Toñita.

A la mitad del plazo fatal, aspirantes como Valenzuela se veían preocupados por el avance en la recolección de las firmas, decía que "era un trabajal de sol a sol" y solo una semana de la fecha fatal dijo ante una reunión que sostuvo con integrantes de "ciudadanos vigilantes" a una pregunta que le hice al respecto, "estamos muy apretados, vamos justo, pero creo que sí lo vamos a lograr". Faltando unos días para el vencimiento del plazo, arreciaron la recolección de firmas. Meléndez, decía tener funcionando alre- 
dedor de diez mesas receptoras y veía difícil, pero no imposible alcanzar la meta, justo, faltando una semana recibió una buena cantidad de firmas de un grupo de pastores evangélicos, lo cual levantó el ánimo y la expectativa de llegar a la cantidad "mágica". También evidenciaba dificultades como:

Algunos de los módulos que tenemos funcionan durante una semana, entendemos que son voluntarios y luego ya no los pueden atender y tenemos que buscar otros voluntarios y bueno, a veces hemos recibido el reclamo de algunas personas que quieren firmar por nosotros y acuden a los módulos que hemos difundido que están instalados en tal o cual parte y resulta que no hay nadie y bueno, no tenemos maneras de obligarlos a que estén allí, no podemos hacer más (Javier Meléndez, entrevista 22 de febrero de 2016).

El aspirante Andrés Carbajal Casas, exmilitante del PRI, excandidato de Morena a una diputación, recientemente se registró bajo la planilla "Yo decido ser independiente"; Alejandro Ramírez Guerrero, postulado por la asociación civil "Vamos de Corazón por Juárez" expanista y es funcionario municipal. En especial, estos dos últimos candidatos se manejaron con bajo perfil mediático, tampoco se les vio en lugares públicos masivos o estratégicos. Carbajal Casas, en la recta final, apareció apoyando a Armando Cabada.

En la entrega de los paquetes con el contenido de las firmas y sus respectivas fotocopias Armando Cabada entregó el 8 de marzo 26 cajas que contenían 88 mil 610 firmas. También ese día se presentó el médico Valenzuela, pero únicamente entregó los formatos, quedando pendiente de llevar toda la evidencia en físico.

Alejandro Ramírez, el aspirante a candidato independiente entregó el 12 de marzo ante la Asamblea Municipal Electoral, la cantidad de 33947 firmas, también dijo, que en dichas firmas se cubrió un $56 \%$ de las secciones electorales y el $2 \%$ de cada sección (Salvador Castro, El Diario de Juárez, 12 marzo 2012). Edna Lorena Fuerte, a punto de vencer el plazo, entregó en la AME 45427 firmas, correspondientes a 720 secciones electorales, con más de un $3.5 \%$ de firmas por sección electoral (http://netnoticias.mx/elecciones/201603-13-6b7549b6/, consultado el 13 de marzo de 2016). 
Finalmente, solamente tres de los siete aspirantes pudieron alcanzar el objetivo de reunir las 29 322. Los otros cuatro no pudieron con este gran obstáculo, se sintió una gran frustración en algunos de ellos, como fue el caso del médico Valenzuela. Hicieron un gran esfuerzo, metieron recursos económicos propios y le dedicaron muchas horas tratando se contribuir para la obtención del anhelado registro.

\section{LAS PRECANDIDATURAS INDEPENDIENTES, ¿EL INICIO DE UN EJERCICIO DE PRIMARIAS?}

Los procesos de selección de candidatos mediante un proceso de competición entre dos o más planillas al interior de los partidos políticos, cada vez son más escasos. Esa práctica democrática de elecciones primarias se dio de manera muy importante con la apertura de las candidaturas independientes, más allá de que se hayan quedado en el camino por los obstáculos impuestos en la Ley Electoral por disposición de los partidos políticos, fue una situación que favoreció la vida democrática de región.

El ambiente, búsqueda de firmas por parte de planillas de diferente origen y composición social, económica y política. Ese acercamiento hacia la ciudadanía buscando el refrendo de esta para competir por el gobierno de la ciudad, fue lo más parecido al proceso anglosajón que llevan a cabo los norteamericanos en la ciudad de El Paso Texas cada dos años. En la vecina ciudad, se desarrollan las elecciones para el gobierno de The City, sin partidos políticos y la búsqueda de registro de candidatos se da mediante un proceso de elecciones primarias con características muy similares a las que se dieron en esta primera participación de precandidaturas independientes.

Como un primer ejercicio para ensanchar los cauces democráticos, sería pertinente en ir pensando en que los procesos electorales municipales se realizaran bajo lo modalidad de candidaturas independientes, pero no como se desarrollan en la actualidad, mediante planillas, sino, separando la elección de alcalde y regidores. Estos últimos, deben ser elector por distrito municipal y representar a los sectores geográficos y sociales de la ciudad, 
para avanzar a una representación más comprometida con los diferentes entornos y necesidades.

\section{LA CANDIDATURA INDEPENDIENTE DE ARMANDO CABADA}

Así empezaba su campaña el candidato independiente por el municipio de Ciudad Juárez:

Hoy inicia esta historia, esta historia que ha sido difícil, que ha tenido muchísimos obstáculos, pero que todos los hemos logrado superar, quiero primero dar gracias a Dios, porque nos ha dado la fortaleza, pero también la tranquilidad, nos ha dado inteligencia para poder llevar este proyecto, que no ha sido nada sencillo. Gracias a muchas personas que han creído en este proyecto, que inició en la navidad de 2015, dar gracias por iniciar una empresa, para la cual tengo la capacidad, que tengo muchos amigos, no fue fácil iniciar con este proyecto, lo que sí fue fácil fue encontrar tantas y tantas voluntades que quieren hacer lo mismo, que queremos una mejor ciudad, permítanme decir una frase que he traído atorada, "este 5 de junio hay que votar por el cambio, hay que votar por los independientes, votar por Armando Cabada, porque es el único que habrá de ofrecer y de cumplir con los anhelos de todos, una mejor ciudad, segura, bonita funcional, esto lo habremos de hacer a partir del 5 de junio".

El empresario y comunicador Armando Cabada, fue el único de los siete precandidatos que sorteó el primer obstáculo, y eso le valió para estar en la planilla. Su arranque se dio con una conferencia de prensa, la cual estuvo abarrotada por gente de su equipo, familia, algunos ciudadanos interesados en su campaña y mucha cobertura mediática de prensa, radio y televisión. Lo primero que hizo, fue presentar los 12 puntos ejes, de su propuesta de gobierno y que se pueden resumir de la siguiente manera:

1. No más baches, habremos de aplicar polímero, como el que se aplica en ciudades tan importantes como el Distrito Federal, el método, la diferencia es que estos polímeros duran mucho más. 
2. Cero corrupciones cuentas claras, transparencia total en mi gobierno. Publicaremos en tiempo real las licitaciones a contratos, servicios de obra. No más moches en la asignación de obra u obras municipales. Hay leyendas urbanas que todos conocemos que datan del 2004. Nunca más pedir cohecho, no más moches, conmigo no cuenten para eso.

3. Crearemos el Instituto Municipal de Seguridad Pública, esta es una propuesta hecha pública y hay que darle el reconocimiento al Plan Estratégico de Ciudad Juárez, en donde se decidirán entre otras cosas, lo siguiente: la selección y evaluación del titular de seguridad pública y el de tránsito, implementaremos los controles de confianza y corrupción a los cuerpos de policía y de tránsito. No más revisiones de rutina, acabaremos con los abusos policiacos, nunca más los van a detener porque se le ocurrió al policía o porque se le ocurrió al agente de tránsito. La responsabilidad de la policía la asumo yo y estoy absolutamente con la policía única, en Juárez somos suficientemente maduros para saber qué es lo que nos duele, la corrupción se tiene que eliminar para tener una policía confiable que nos cuide a todos por igual.

4. La Junta de Aguas para Juárez, cerraré la caja chica de las campañas del PRI. Iniciaremos con el gran proyecto de drenaje pluvial, es injusto dejar que Juárez se inunde.

5. Que tus impuestos regresen y se inviertan en Ciudad Juárez, exigiremos la redistribución de las participaciones estatales, los juarenses aportamos mayor cantidad de recursos y recibimos mucho menos de lo que recibe la capital del estado. Juárez merece estar más bonita que la propia capital.

6. Cabildo abierto a la ciudadanía, debo reconocer que esta también es una propuesta de Plan Estratégico de Ciudad Juárez. Habrá votación de la ciudadanía en tiempo real, mediante las redes sociales.

7. Alumbrado público, pero sin transas. Nosotros atenderemos el tema de manera eficiente, gradual. Lo haremos desde el municipio con los ahorros que son aproximadamente 17 millones y lo haremos en esas cuevas que son el lugar más oscuro y que 
son donde están atacando a nuestros obreros, a nuestros empelados de maquiladora, a nuestras mujeres, ahí empezaremos.

8. Estableceremos estancias infantiles para niños de 0 a 4 años, implementaremos programas para aprender en casa a evitar el bullying.

9. Una casa deportiva cerca de ti, un centro comercial, una cancha deportiva de usos múltiples en la parte del estacionamiento que ellos designen (Smart), para fomentar así la competencia deportiva entre los mismos centros comerciales, a cambio de lo anterior el municipio deducirá una porción del impuesto predial. "Una cancha en su camino". Rescate del Chamizal mediante un proyecto integral que permita recuperarlo para todos y darle accesibilidad para quien quiera practicar algún deporte.

10. Apoyo a los comerciantes, no más mordidas a inspectores, permiso de comercio sin costo por los próximos dos años para el comercio informal. Para el comercio formal, expedición rápida de las licencias y por internet. Para los negocios establecidos, primero la licencia y después la verificación, por eso promoveremos la apertura de nuevos negocios, por cierto, no más requisito del Sistema de Información Empresarial Mexicano (SIEM) para obtener una licencia de funcionamiento.

11. Educación y cultura, el auditorio Benito Juárez sin costo para los artistas locales, se apoyara el arte urbano, mediante el programa arte en las calles.

12. Apoyo a los adultos mayores, atención integral para ellos, hacerles justicia.

Estos 12 puntos, alrededor de 8 millones de pesos, el apoyo de su medio de comunicación y otros más, lo posicionaron rápidamente en la preferencia de la ciudadanía. Realizó una campaña intensa, lo hizo en sectores estratégicos, contó con el apoyo de cámaras empresariales, sectores que tradicionalmente favorecían al PRI y con la experiencia de priístas que fueron relegados

Se montó una campaña de guerra sucia contra él, sin embargo no funcionó, como tampoco, los intentos de sacarlo de la jugada mediante demandas en su contra por diversos motivos, eso, lo victimizó y el apoyo popular creció. Pero, es importante resaltar, 
que su campaña, no solo se fortaleció en las zonas más marginadas, también un amplio sector de las clases medias lo apoyaron.

Tuvo el cierre de campaña más concurrido de todos, había alrededor de 40 mil personas, además del ambiente que se percibía. Se congregaron familias completas, el espacio que se escogió para llevar a cabo el cierre, fue insuficiente. El día de la jornada electoral, se vio una intensa movilización de personas votando en las casillas de las diferentes áreas de la ciudad. Hacía tiempo que no se veía esa expresión y esa manifestación en una jornada electoral, lo que auguraba buenos signos, se había planteado la hipótesis de que si salía la gente a votar, el resultado sería adverso para el PRI y, efectivamente, así fue.

El candidato independiente obtuvo 209782 votos; le siguió el PRI-PVEM-PT-PNA, con 107 378; en un alejado tercer lugar, el PAN con 62 606; MORENA con 18 167; Encuentro social, 8123; PRD, 4719. Por lo que se puede apreciar con estos resultados, el triunfo para el candidato independiente fue arrollador.

Asimismo, se dieron dos impugnaciones posteriores a la jornada electoral, ambas por parte del Partido Revolucionario Institucional (PRI), ninguna de ellas prosperó y el candidato electo por la planilla Independiente tomará protesta en el mes de octubre de 2016. Por parte de la ciudadanía, existen amplias expectativas de que sea un gobierno que cumpla con las propuestas de campaña. Esta vez, se apostó por un gobierno sin partido político en el municipio de Juárez.

\section{CONCLUSIÓN}

La Reforma Electoral que inició en 2012 y concluyó en 2014 generó una enorme expectativa en torno a las candidaturas independientes, mismas que posibilitan la participación electoral de un ciudadano sin militancia partidista. En ese sentido se entiende la independencia de estas candidaturas, en que no tengan que estar avaladas con la rúbrica de un partido político. En los hechos, la gran mayoría de quienes han solicitado registro para contender por esta vía, tienen antecedentes de una, dos o más militancias anteriormente. Ahora, no necesitan involucrarse en un instituto 
político al que deban subordinarse, pueden intentar desde fuera de estos buscar posiciones políticas surgidas de procesos electorales populares.

Una condición que ha quedado manifiesta en los diferentes escenarios que se han realizado elecciones incluyendo las candidaturas independientes, es la cuestión económica. Quien tenga recursos para realizar la recolección de firmas, pagando un amplio equipo que se encargue de recabarlas abarcando toda una estrategia logística, puede participar con probabilidades reales de sortear los obstáculos legales que se han puesto.

En el plano nacional, logró entrar un personaje con un capital político construido por su padre y mantenido por él, Clouthier, ingresó al Congreso Nacional en la Cámara de Diputados, siendo el primero en hacerlo por esta vía. A nivel local, un joven de Zapopán, Jalisco, Pedro Kumamoto, hizo lo mismo en el Congreso local. En Nuevo León, un alcalde fue electo por esta vía; y la cereza del pastel, la representó un expriísta, ganando nada más y nada menos que la gubernatura del Estado, el denominado "Bronco", obtuvo de manera sorpresiva el poder ejecutivo de una de las entidades más ricas del país.

Esta situación prendió los focos rojos en las entidades que tendrían procesos electorales en 2016, entre ellas Chihuahua, entidad que armaría una reglamentación electoral conocida como anti-Bronco, dejando fuera del proceso a quienes habían tenido una militancia partidista inferior a tres años, dicha reforma fue diseñada para que no contendieran priístas inconformes con su propio proceso interno de selección o exmilitantes que recién hubieran dejado las filas del partido. Esta ley, terminó afectando a suspirantes de todos los partidos políticos. También, esta ley, fue diseñada para hacer muy difícil el acceso a la categoría de candidatos. Sin embargo, este apartado fue echado abajo por el tribunal electoral, el problema fue, que ya habían pasado los tiempos de registro para las precandidaturas.

En la experiencia de Ciudad Juárez, de siete planillas que se inscribieron como aspirantes al ayuntamiento, solamente tres lograron reunir la cantidad de firmas, de estos, a dos se les anularon las suficientes, de tal suerte que aún sin dar resultados definiti- 
vos, solamente se aceptó una planilla en la siguiente etapa, que sería de registro y así poder contender en el proceso electoral con esa categoría.

El único aspirante que se mantuvo en el proceso, fue un empresario de los medios de comunicación local. Su trayectoria de 27 años en el noticiero más visto de la Frontera juarense, la cobertura mediática y los recursos económicos, políticos y jurídicos de los que dispone le ha permitido mantenerse. Ha tenido impugnaciones y se han resuelto amonestaciones de los órganos electorales en su contra. Se esperaron hasta el último momento de los tiempos legales para informarle que sí logró cumplir con la totalidad de los requisitos, que son: a) obtener $3 \%$ de las firmas del padrón electoral del municipio, lo que equivale a 29 622; cubrir más de un $50 \%$ de las secciones electorales; y, que las firmas correspondan al $2 \%$ en cada sección.

Esta primera experiencia electoral para las candidaturas independientes en Ciudad Juárez, dejó en claro que todavía hay partidocracia para rato, está debilitada, pero aún se mueve. Además, goza del apoyo de los órganos electorales. La única candidatura independiente que no aceptó los recursos públicos para la campaña que eran de alrededor de 28 mil pesos moneda nacional.

El Estado de Chihuahua, pasa por la peor corrupción de su historia, eso contribuyó a que se diera un voto diferenciado y un voto útil contra el PRI. Los triunfadores a nivel local, fueron los candidatos del PAN y los independientes de dos importantes ciudades de la entidad. Juárez, que fue ganada con casi el doble de los votos con respecto a su más cercano competidor, el desprestigiado candidato del PRI; y el independiente municipal en Parral, que fue una situación parecida a la de Ciudad Juárez. La primera elección con candidatura independiente municipal en esta nueva modalidad, fue favorecida ampliamente por el voto popular. El gobierno del Estado, estará gobernado por el PAN y en el Congreso, por primera vez, el PRI no tendrá mayoría. 


\section{BIBLIOGRAFÍA}

Campos, Gonzalo Santiago (2014). Las candidaturas independientes en México. Revista Derecho del Estado núm. 33, julio-diciembre de 2014, pp. 65-99.

Fuentes Díaz, Vicente (1969). Los partidos políticos en México. México: Altiplano.

González Casanova, Pablo (1975). La democracia en México. México: Era.

Instituto Nacional Electoral (2015).

Instituto Estatal Electoral de Chihuahua (2015).

Partido Acción Nacional.

Partido de la Revolución Democrática.

Partido Popular Socialista.

Partido Revolucionario Institucional.

Rodríguez Araujo, Octavio (1989). La reforma política y los partidos políticos en México. México: Siglo XXI Editores.

Sartori, Giovanni (2002). Partidos y sistemas de partidos: marco para un análisis. Madrid: Alianza.

\section{Páginas de Internet}

http://democraciamexico.blogspot.mx/2006/candidaturas-independientes-en-el.html, consultado el 5 de marzo de 2016.

http://www.fusda.org/Revista 13-2AlejandroChanona.pdf, consultado el 5 de marzo de 2016.

http://www.adnpolitico.com/opinion/2012/analisis-una-mirada-a-las-

candidaturas-independientes, consultado el 6 de marzo de 2016.

http://podemos.info/Podemos.info, consultado el 7 de marzo de 2016.

http://www.diputados.gob.mx/museo/s_nues8.htm, consultado 12 de marzo de 2016.

http://timingpolitico.com/archivos/4713, consultado el 25 de febrero de 2016.

http://www.oem.com.mx/elmexicano/notas/n4074643.htm, consultado

el 27 de febrero de 2016. 
NOLBERTO Acosta

\section{Entrevistas}

Antonia Hinojos Hernández, realizada el 23 febrero de 2016.

Javier Meléndez Cardona, realizada el 22 febrero 2016.

Arturo Valenzuela Zorrilla, realizada el 25 febrero 2016. 